Digital Press Social Sciences and Humanities

Représentation des relations franco-maghrébines dans le roman Apocalypse bébé : apprendre la culture françaisemaghrébine à travers des textes littéraires

Novi Kurniawati

Proceeding of Conférence internationale sur le français 2018

Joesana Tjahjani, Merry Andriani, Sajarwa, Wening Udasmoro (eds) 


\title{
Représentation des relations franco-maghrébines dans le roman Apocalypse Bébé : apprendre la culture française- maghrébine à travers des textes littéraires
}

\author{
Novi Kurniawati \\ Universitas Negeri Semarang, Semarang, Indonesia \\ e-mail : noviekurnia25@gmail.com
}

\begin{abstract}
Résumé
L'apprentissage d'une langue étrangère ne peut être séparé de la littérature et de la culture. Une des définitions de la littérature est un reflet de la société, donc à travers la littérature, nous pouvons connaittre l'image réelle de la société ainsi que la culture. De plus, en connaissant les cultures étrangères des pays dont nous apprenons la langue, nous pouvons non seulement lire, mais aussi comprendre les problèmes qui apparaissent dans les textes étudiés. De même, la culture française ne peut pas être séparée de la culture maghrébine. Les deux cultures se complètent, devenant plus tard le contenu de diverses histoires littéraires, littéraires françaises et francophones. La relation entre les deux cultures fait également partie du contenu du roman intitulé Apocalypse Bébé de Virginie Despentes. A travers ce roman, nous pouvons voir une image de la relation entre la France et le peuple maghrébin dans leur vie sociale. Ainsi, en tant qu'apprenant du français, nous connaissons non seulement la culture française à travers les manuels publiés par les éditeurs français, mais reconnaissons aussi la culture francophone associée à la France à la fois directement et indirectement. Par conséquent, le texte littéraire intitulé Apocalypse Bébé peut être une source alternative d'apprentissage du français, non seulement en termes de langue attachée au vocabulaire et à la grammaire, mais aussi pour connaître la culture française et francophone afin que les étudiants connaissent leur relation pour comprendre et analyser les œuvres littéraires
\end{abstract}

\section{Mots-clés}

français, francophone, culture, texte littéraire

\begin{abstract}
Learning a foreign language cannot be separated from literature and culture. One of the definitions of literature is a reflection of society; so through literature we can know the real image of society as well as the culture. Moreover, by knowing the foreign cultures of the countries from which we learn the language, we can not only read, but also understand the problems that appear in the texts studied. Similarly, French culture cannot be separated from Maghreb culture. The two cultures complement each other, later becoming the content of various literary, French literary and Francophone literary. The relationship between the two cultures is also part of the content of Virginie Despentes' novel Apocalypse Bébé. Through this novel, we can see an image of the relationship between France and the Maghreb people in their social life. Thus, as a learner of French, we could know not only French culture through the textbooks published by French publishers, but also recognize the French culture associated with France both directly and indirectly. Therefore, the literary text entitled Apocalypse Bébé can be an alternative source of learning French, not only in terms of language attached to vocabulary and grammar, but also to know French and Francophone culture so that students know the relationship for understanding and analyzing literary works.
\end{abstract}

\section{Keywords}

French, Francophone, culture, literary text 


\section{Introduction}

L'apprentissage des langues étrangères est très étroitement lié à la culture du pays où nous apprenons la langue afin que nous puissions utiliser la langue en fonction du contexte. Par conséquent, dans cet apprentissage, nous ne nous concentrons pas uniquement sur le vocabulaire et la grammaire, mais également sur la culture qui est souvent évoquée à travers des œuvres littéraires. L'un des objectifs de l'enseignement d'une langue étrangère est la connaissance de la littérature et de la culture du pays. Selon Godard (2015, p. 49) dans l'enseignement du français langue étrangère (FLE), la littérature peut devenir une voie d'accès à la culture anthropologique. Georges Lüde, Beat Munch et Claude Gauthier $(1994, \mathrm{p}$. 105) expliquent que l'enseignement de la civilisation comme collection de monuments qui a été remis en question au profit d'une didactique de la culture, incluant savoirs socioculturels, schématisations discursives et pratiques culturelles. Alors, les œuvres littéraires ont le rôle important dans l'enseignement du français langue étrangère qui peut donner des connaissances du monde.

Une des définitions ou des fonctions de la littérature est comme un reflet ou miroir de la société. En tant qu'un reflet ou miroir de la société, la littérature est comme forme d'art imaginatif qui est pris de la réalité et la nature (Ratna, 2007, p. 306). C'est-à-dire, les histoires dans les œuvres littéraires viennent de la réalité qui est modifié par les écrivains en utilisant l'imagination. Selon Faruk (2012, pp. 54-55) comme représentation, la littérature peut construire le monde imaginaire, un environnement d'une interaction imaginaire qui reflète un modèle de l'interaction qui existe dans le monde réel. Ainsi que le roman français intitulé Apocalypse Bébé de Virginie Despentes (2010) avec les personnages qui construisent la relation inséparable les uns les autres entre la France, le français et les maghrébins.

D’après l'Institut national de la statistique et des études économiques (dans Belhaiba, 2014, p. 36) la population des maghrébins en France montre une augmentation significative chaque année. On peut donc dire que ce phénomène devient une étude importante à étudier parce que cela fait partie de la réalité en France qui donne des effets dans le domaine culturel. C'est pourquoi les apprenants de la langue française ont aussi besoin de savoir cette connaissance pour qu'ils puissent bien comprendre non seulement la langue française mais aussi le phénomène culturel qui se passe en France, le pays où on apprend la langue.

Selon Godard (2015, pp. 139-140) la présence de la littérature dans les méthodes de FLE des éditeurs français est encore limitée. Elle dit que sur 55 manuels étudiés, 20 ne font apparaître aucun texte littéraire, ainsi que les méthodes qui font apparaitre le phénomène de cette relation franco-maghrébine. Alors, cet article a pour but de donner une petite explication sur la représentation de la relation francomaghrébine dans le roman Apocalypse Bébé par Virginie Despentes qu'on peut utiliser comme un médium culturel qui est inséparable de langue française.

\section{Résultats et discussions}

\subsection{Pourquoi le texte littéraire devrait-il être dans l'apprentissage de FLE ?}

Le texte littéraire est un genre très rarement utilisé dans les classes de français langue étrangère. D'après Roux (2016) une des causes de cette absence est la croyance qui veut dire que les textes littéraires ne peuvent s'adresser qu'à des étudiants de niveau avancé. Le texte littéraire a tout son intérêt dans la classe de français langue étrangère et dès les premiers niveaux d'apprentissage. Cependant, pour être motivant et efficace, son utilisation doit d'une part préciser ses objectifs pédagogiques et d'autre part doit respecter un certain nombre de principe didactique.

L'utilisation de la littérature dans la classe de français langue étrangère est en développement pour plusieurs raisons (Lettres et Langue Français, n.d.). La littérature est devenue moins populaire dans l'enseignement des langues et l'apprentissage parce qu'on commence à se concentrer sur l'utilisation fonctionnelle du langage, le vocabulaire et la grammaire. Cependant, le rôle de la littérature dans la classe de FLE a été réévalué. Selon Christian Puren et Isabelle Gruca (dans Godard, 2015, p. 14) les principales méthodologies scolaires, qui se sont succédé jusqu'au milieu du XXe siècle, sont fondées sur une conception assez proche des relations entre langue, littérature et culture et utilisent le texte littéraire comme support principal des leçons en considérant qu'il est à la fois un réservoir de formes, un modèle de langue et un concentré de culture étrangère. On utilise le texte littéraire parce que la littérature est 
assimilée à la culture, dont elle est considérée comme l'accomplissement artistique dans le domaine langagier et qu'on s'intéresse peu, alors, aux formes anthropologiques de la littérature, qui se donnent à voir dans des pratiques sociales. Ensuite, la littérature, en tant qu'art du langage, est placée au sommet d'une hiérarchie établie dans la langue entre d'un côté les formes populaires et le langage courant, jugés moins dignes d'être enseignés, et de l'autre côté, les formes savantes valorisées par la culture scolaire centré sur l'écrit. En d'autres termes, les textes littéraires sont toujours liés à la culture et ne peuvent être séparés les uns des autres. En lisant des textes littéraires, nous pouvons apprendre non seulement la langue, mais nous pouvons aussi ajouter des connaissances sociales et de l'anthropologie, afin que nous puissions connaître et d'entrer dans la langue et la culture de l'autre et de restructurer notre propre identité.

Cervera (dans Allam-Iddou, 2015, pp. 100-101) dit que le texte littéraire comme une expression, un regard fragmentaire porté sur un modèle culturel. C'est en ce sens que nous envisageons l'apprentissage d'une langue étrangère. Puisqu'apprendre une langue étrangère ce n'est pas seulement étudier la langue, il convient d'établir un parallèle entre la découverte d'une culture et l'apprentissage d'une langue, en somme, de mettre en évidence cette culture partagée. L'apprentissage de la langue étrangère implique donc la découverte de nouvelles pratiques culturelles et de nouvelles valeurs. Alors, l'utilisation des textes littéraires dans la classe de français langue étrangère peut être un espace favorable de connaître et de rencontrer d'autres cultures. Donc, cela peut aussi sensibiliser les apprenants à la différence et à la diversité, stimuler et développer chez eux le côté intellectuel.

Selon Allam-Iddou (2015, p. 101) l'objectif de la démarche interculturelle des textes littéraires dans la classe de FLE consiste à apprendre aux apprenants de travailler sur les préjugés, les représentations et les stéréotypes préconçus de la culture étrangère. De ce fait, on peut dire que les textes littéraires ont beaucoup de connaissances utiles aux apprenants de langues, de sorte qu'ils peuvent les intégrer dans des activités d'apprentissage de langues étrangères.

\subsection{Quels sont les défis?}

Les textes littéraires peuvent présenter aux enseignants et aux apprenants un certain nombre de difficultés. Le premier, la sélection de texte peut devenir une tache pour les enseignants parce qu'ils doivent choisir les textes présentant une pertinence et un intérêt pour les apprenants. Le deuxième, la difficulté linguistique parce qu'on doit adapter les textes qui au niveau de compréhension des élèves. Le troisième, la longueur des supports pédagogiques peut aussi devenir une difficulté parce que les textes plus courts peuvent être plus faciles à utiliser dans les activités d'apprentissage, mais les textes plus longs fournissent plus de détails contextuels et le développement du caractère et de l'intrigue. Ensuite la difficulté culturelle où on ne peut pas choisir les textes qui sont tellement culturellement denses que les personnes extérieures se sentent exclues de la compréhension d'un sens essentiel.

Pour faire face aux défis présentés ci-dessus, il y a plusieurs choses à faire. Avant d'utiliser les textes littéraires dans la classe de FLE, on doit poser une série de questions pour évaluer la pertinence des textes pour un groupe particulier d'apprenants, par exemple le sujet est-il susceptible d'intéresser les élèves ? Le niveau de langue est-il approprié au niveau des apprenants ? Est-ce la longueur du texte est appropriée au temps disponible ? Est-ce qu'il nous faut beaucoup de connaissances culturelles ou littéraires? Les enseignants peuvent aussi exploiter des textes littéraires de nombreuses façons dans la classe de français. Le travail en classe avec des œuvres littéraires peut comporter des tâches de prélecture, des travaux interactifs sur le texte et des activités de suivi. La lecture devient le premier passage obligatoire pour initier les apprenants à l'utilisation du texte littéraire. La lecture de ce texte peut permettre aux apprenants de mobiliser leur propre réalité, expérience et représentations. Les enseignants peuvent présenter le sujet ou le thème du texte, pré-enseigner les éléments essentiels du vocabulaire et utiliser des tâches de prédiction pour susciter l'intérêt et la curiosité des étudiants. On peut également aider les élèves à apprécier les façons dont les écrivains utilisent le langage pour obtenir des effets particuliers.

\subsection{La France, les français et les maghrébins dans le roman Apocalypse Bébé: voir les relations culturelles à travers du texte littéraire}

Apocalypse Bébé est un roman français qui contient une histoire complexe entre le genre et la vie sociale des français. Pourquoi le genre ? Parce que ce roman parle de la vie des lesbiennes qui existe dans la société française. Despentes, en tant qu'un écrivain, elle ne parle pas seulement du genre dans son œuvre, 
mais aussi la vie sociale française à travers des personnages du Maghreb qui va être une discussion dans cet article. Elle a mis le problème de l'identité des lesbiennes et des personnages du Maghreb dans la même position dans la société française.

Ce problème de l'identité devient important dans la pratique sociale en France parce qu'il porte indirectement des séparations entre des citoyens. Par exemple, la séparation géographique où le centreville est identiquement aux Français et la banlieue est identiquement comme les résidences des maghrébins. Cette séparation-là qui ensuite crée un stéréotype où les gens habitant dans le centre-ville sont des Français qui ont les habitudes élites, luxes et soignées. Ce sont comme manger de la marque dans des assiettes en porcelaine, se laver les mains avant de se mettre aux devoirs, avoir un sac en peau bordeaux, avoir une habitude intellectuelle ayant vingt livres sur étagère et bafouiller trois mots d'anglais. La représentation de cette condition a été montrée par Virginie Despentes à travers les personnages qui s'appellent Louisa, Valentine et la famille de Loraine et elles sont françaises. C'est bien sûr différent avec la famille de Louisa qui est du Maghreb et vit à la banlieue avec des habitudes simples et désordonnées (Kurniawati, 2016, p. 64). Cela peut causer de la distance dans la pratique sociale entre les français et les maghrébins qui ont déjà la nationalité française.

Outre la séparation géographique ci-dessus à travers les discours du centre-ville et de la banlieue, ce roman montre également le phénomène des Français qui ont la légitimité d'être racistes face à une société différente de leur position. Cette condition a été représentée par Virginie Despentes à travers du personnage Louisa qui est raciste comme les autres français quand elle était à Barcelone. Mais, elle ne peut pas faire comme ce qu'elle a fait à Barcelone quand elle est à Paris parce qu'elle a du sang d'origine maghrébine (Kurniawati, 2016, p. 67). D'un côté, devenir Français est une fierté d'être en position supérieure. D'un autre côté, devenir Français peut créer ses propres peurs à propos des règles et des normes qui le suivent. Ainsi, nous pouvons dire que ces conditions ne peuvent être séparées du pays qui a le pouvoir d'établir des règles liées à l'identité de leurs citoyens.

Dans ce cas-là, l'Etat français a une influence considérable sur l'identité des populations du Maghreb vivant en France. À travers le récit des figures de Yacine et de Louisa, deux descendants du Maghreb, Despentes a tenté de montrer comment l'État a contribué à la formation de l'identité de ses citoyens. Le contrôle exercé par l'Etat dont l'un des objectifs est de maintenir 'l'âme de Français' comme symbole des citoyens. Ce contrôle est fait par l'Etat à travers l'enseignement du français qu'on donne à l'école en tant que cours obligatoire. Ce phénomène a été montré par Despentes par le personnage Vanessa qui vient de la famille maghrébine. Elle essaie de parler le sarcellois, un mélange de rebeu et de français incorrect, avec ses amis dans la communauté. On peut dire que cela peut être une forme de résistance aux règles de l'Etat et de détermination de l'identité en tant que maghrébins. Cependant, la résistance se heurtera toujours à la réglementation de l'État qui oblige les écoles à faire enseigner le français correctement (Kurniawati, 2016, p. 70). Cette explication montre que les maghrébins ayant la nationalité française essaient d'avoir leurs propres caractéristiques qui le distinguent des Français, mais cet effort sera toujours surveillé par l'État afin qu'ils ne soient pas séparés de l'identité en tant que citoyens français.

Les phénomènes évoqués ci-dessus ne se retrouvent pas dans les méthodes du français langue étrangère qui est souvent utilisé dans l'apprentissage du français, alors qu'en tant qu'apprenant du français, ils ont vraiment besoin de connaître les informations socioculturelles qui se développent dans le pays où la langue est apprise. Par conséquent, nous devons utiliser un texte littéraire qui peut servir de source d'information culturelle. La relation entre le français et les maghrébins illustrée dans le roman AB peut être considérée comme une réflexion sociale française vivant à Paris. Cependant, nous devons également nous rappeler qu'Apocalypse Bébé est l'un des œuvres littéraires qui sont imaginaire. Nous devons donc choisir précisément les informations qui seront transmises aux apprenants lors de l'utilisation de textes littéraires dans la classe FLE.

\section{Conclusion}

Le texte littéraire peut être une alternative dans l'apprentissage de FLE. En plus de contenir des éléments de langue, les textes littéraires deviennent également une source de connaissances socioculturelles, de sorte que les apprenants peuvent développer leur compréhension d'autres cultures, prendre conscience de la différence et développer la tolérance et la compréhension.

Les maghrébins font partie intégrante de la société sociale française. Cependant, le phénomène est très rare à trouver dans les méthodes créées par les éditeurs français. C'est pourquoi nous avons besoin d'utiliser des textes littéraires pour voir les relations qui se produisent entre les maghrébins et les 
français, surtout en France afin que nous puissions connaître la culture du pays où nous apprenons la langue et nous pouvons utiliser la langue selon le contexte socioculturel en développement dans le pays.

\section{Références}

Allam-Iddou, S. (2015). De l'usage des textes littéraires comme outil didactique pour

l'enseignement/apprentissage du Français Langue Étrangère. Synergies Chili, (11), 95-103. Retrieved from https://gerflint.fr/Base/Chili11/allam-iddou.pdf

Belhaiba, A. (2014). Le langage des jeunes issus de l'immigration maghrébine à Bordeaux : pratiques, fonctions et représentations. Université Michel de Montaigne-Bordeaux III, Bordeaux. Retrieved from http://www.theses.fr/2014BOR30015/document

Despentes, V. (2010). Apocalypse Bébé. Paris: Édition Grasset \& Fasquelle.

Faruk, H. T. (2012). Pengantar Sosiologi Sastra dari Strukturalisme Genetik sampai Post-modernisme.

Yogyakarta: Pustaka Pelajar.

Godard, A. (2015). La littérature dans l'enseignement du FLE. Paris: Les Éditions Didier.

Kurniawati, N. (2016). Politik Performatif dalam Novel Apocalypse Bébé Karya Virginie Despentes :

Konstruksi Identitas Lesbian melalui Sastra. Universitas Gadjah Mada, Yogyakarta.

Lettres et langue Francais. (n.d.). didactique des textes littéraires en classe de FLE.

Lüde, G., Munch, B., \& Gauthier, C. (1994). l'évolution de la didactique des langues (1968-1988. In D. Coste

(Ed.), l'évolution de la didactique des langues (1968-1988) (pp. 100-116). Paris: Hatier/Didier.

Ratna, N. K. (2007). Sastra dan Cultural Studies : Representasi Fiksi dan Fakta. Yogyakarta: Pustaka Pelajar. Roux, P.-Y. (2016). Enseigner le FLE à partir de textes littéraires (du niveau A2 au niveau B2). Asie du Sud :

Université régional-BELC Inde. 